Reprinted from The Journal of Chemical Physics, Vol. 45, No. 5, 1505-1514, 1 September 1966

Printed in U. S. A.

\title{
Viscoelasticity of Networks Consisting of Crosslinked or Entangled Macromolecules. I. Normal Modes and Mechanical Spectra
}

\author{
A. J. СномpғF \\ Scientific Research Staff, Ford Motor Company, Dearborn, Michigan \\ AND \\ J. A. DUISER \\ Central Laboratory T.N.O., Delft, The Netherlands
}

(Received 7 February 1966)

\begin{abstract}
A molecular theory is developed to describe quantitatively the mechanical behavior of entanglement networks of linear, randomly coiling molecules. The theory is based on the model of Rouse for a single molecule and is a generalization of the theory of Duiser and Staverman for chemically crosslinked networks.

A new model for an "entanglement point" is suggested, which accounts for the frictional force due to the velocity difference between two entangled molecules at that point. This leads to a modification of the diffusion equation in the Rouse theory.

It turns out that the relaxation spectrum of a network corresponds to that of an assembly of "decoupled" equivalent molecules in which the ends of some submolecules have a mobility which is smaller by a slip factor $\delta$. This parameter may have values from zero (crosslinked molecules) to unity (free molecules in dilute solution).

The relaxation spectra of the decoupled equivalent molecules can be obtained by applying a property of the Sturm sequence in a computer program, without actually solving for the eigenvalues of the modified matrix in the Rouse diffusion equation.

Application of the theory to experimental data yields the number of elastically effective network chains of an entanglement network by integration of the area under the curve in the long-time region of the relaxation spectrum.
\end{abstract}

\section{INTRODUCTION}

$\mathbf{I}$ $\mathrm{N}$ the literature dealing with the mechanical behavior of polymers and polymer solutions, several theories can be found which attempt to describe this behavior on a molecular basis. ${ }^{1-5}$ In most cases, these theories concentrate on a rather narrow field of interest. Many molecular theories are still restricted to linear viscoelastic behavior of linear, randomly coiling polymer chains. Furthermore they must still be extended in order to explain the long-time regions where entanglements affect the viscoelastic properties. Entanglements

\footnotetext{
${ }^{1}$ A. J. Staverman, in Handbuch der Physik, S. Flügge, Ed. (Springer-Verlag, Berlin, 1962), Vol. 13, pp. 432-451.

${ }^{2}$ L. R. G. Treloar, in Die Physik der Hochpolymeren, H. A. Stuart, Ed. (Springer-Verlag, Berlin, 1956), Vol. 4, pp. 295-372.

${ }^{3}$ J. D. Ferry, Viscoelastic Properties of Polymers (John Wiley \& Sons, Inc., New York, 1961), pp. 151ff.

${ }^{4}$ A. V. Tobolsky, Properties and Structure of Polymers (John Wiley \& Sons, Inc., New York, 1960), pp. 160ff.

${ }^{5}$ A. V. Tobolsky and J. J. Aklonis, J. Phys. Chem. 68, 1970 (1964).
}

are present in concentrated polymer solutions as well as in bulk polymers, whether chemically crosslinked or not. Characterization of such entanglement networks as well as chemically crosslinked, permanent networks involves the determination of the number of entanglements and/or permanent crosslinks. Permanent crosslinks give rise to an equilibrium rubber elastic modulus, which can be viewed as resulting from a number of molecular relaxation times which have become infinite. Entanglements give rise to a series of relaxation times, which are not infinite but very long. Entanglement networks therefore do not show an equilibrium modulus but behave rubber elastically in a certain time scale.

The dearth of precise knowledge in this field, reflected in several controversies in current polymer network theories, provides the main impetus for research in this area. One of the present authors (J.A.D.) has recently ${ }^{6}$ extended the molecular model of Rouse ${ }^{7}$ for linear

\footnotetext{
${ }^{6}$ J. A. Duiser, Ph.D. thesis, Leiden, The Netherlands, 1965.
}

7 P. E. Rouse, Jr., J. Chem. Phys. 21, 1272 (1953). 
polymer molecules to permanent networks and has also considered special cases of temporarily crosslinked networks. The other author (A.J.C.) has extended the Rouse model to entanglement networks, ${ }^{8}$ thereby giving a more general derivation of the relaxation spectrum for crosslinked and entanglement networks. The present cooperation has resulted in a quantitative generalized molecular theory for both crosslinked and entangled networks, by means of the introduction of a slippage parameter between polymer chains, which becomes zero in the case of a permanent network.

The molecular model on which Rouse's theory is based does not always give as close an agreement with experiment as models proposed later, ${ }^{9-11}$ but is easier to modify for the effect of temporary crosslinks due to chain entanglements and for the effect of permanent chemical crosslinks. The reason is that a very important part of the mechanical behavior in Rouse's theory is incorporated in a "mobility-coefficient matrix" which accounts for the mobility at several points in the chain. It is possible to modify Rouse's theory by suitably changing this matrix.

\section{SINGLE-CHAIN PROBLEM}

A few premises, similar to those of Rouse's theory, ${ }^{7}$ are outlined below for an unentangled polymer chain.

A free-draining molecule of randomly coiling, freely jointed segments is considered, as postulated by Debye. ${ }^{12}$ The solution containing the molecule is assumed to be sheared by harmonic motion in the $x$ direction of a $z=$ constant plane. One end of the molecule is chosen as the origin of this coordinate system.

The molecule is thought of as being composed of $N$ equal submolecules of a length, which is rather arbitrary, but which is so chosen that the end-to-end distance of a submolecule follows a Gaussian probability distribution function. The purely viscous resistance of the medium is imagined to be concentrated at the junctions between the submolecules, while the submolecules themselves are supposed to act like ideal elastic entropy springs.

A submolecule with a length which fulfills the above conditions has a rms end-to-end distance given by $\left\langle\boldsymbol{r}_{\mathrm{s}}{ }^{2}\right\rangle^{\frac{1}{2}}=a(q)^{\frac{1}{2}}$, where $q$ is the number of monomer units in the submolecule and $a$ is an effective monomer length.

Each submolecule has its own Cartesian-coordinate system $x_{j}, y_{j}, z_{j}$ parallel to the original system $x, y, z$. The configuration of the entire molecule is then described by a so-called representative point in a $3 N$ dimensional configuration space.

The rate of change of this configuration depends both on the velocity gradient of the solvent and on the

\footnotetext{
${ }^{8}$ A. J. Chompff, Ph.D. thesis, Delft, The Netherlands, 1965.

${ }^{9}$ B. H. Zimm, J. Chem. Phys. 24, 269 (1956).

${ }_{10}$ N. W. Tschoegl, J. Chem. Phys. 39, 149 (1963).

${ }^{11}$ N. W. Tschoegl, J. Chem. Phys. 40, 473 (1964).

12 P. Debye, J. Chem. Phys. 14, 636 (1946).
}

tendency of the molecule to diffuse back to its equilibrium position. Only the latter of the two ratedetermining components is altered in the present treatment.

According to Rouse the average velocity of back diffusion $\left(\dot{x}_{j}\right)_{D}$ of the representative point in this $3 N$ space along the $x_{j}$ coordinate towards its equilibrium position after deformation is found to be

$$
\left(\dot{x}_{j}\right)_{D}=-B\left[-\frac{\partial \mu}{\partial x_{j-1}}+2 \frac{\partial \mu}{\partial x_{j}}-\frac{\partial \mu}{\partial x_{j+1}}\right],
$$

where $\mu$ is the chemical potential of the molecule and $B$ is the average mobility of the junctions between the submolecules. For small displacements from equilibrium $\partial \mu / \partial x_{j}$ is the driving force for the back diffusion in the $x_{j}$ direction; the other differentials in Eq. (1) have an analogous meaning.

The set of $N$ equations for all the $\left(\dot{x}_{j}\right)_{D}$ can be written

where

$$
\dot{\mathbf{x}}_{D}=-B \mathbf{A}_{33} \boldsymbol{\nabla}_{x \mu},
$$

$$
\begin{aligned}
& \dot{\mathbf{x}}_{D}=\left\{\dot{x}_{1 D}, \dot{x}_{2 D}, \dot{x}_{3} D, \cdots \dot{x}_{N D}\right\}, \\
& \boldsymbol{\nabla}_{x}=\left\{\frac{\partial}{\partial x_{1}}, \frac{\partial}{\partial x_{2}}, \cdots \frac{\partial}{\partial x_{N}}\right\},
\end{aligned}
$$

and $\mathbf{A}_{33}$ is the square $N$ th-order matrix

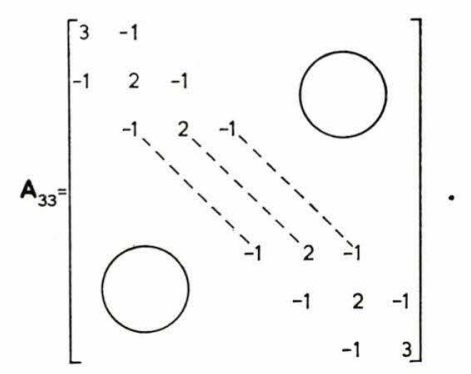

Following Duiser and Staverman, ${ }^{13}$ the mobilities assigned to the two points determining the ends of the free chain are taken as at least twice the average mobility of the submolecules in the middle, since these points are connected to only one submolecule. This accounts for the 3's at the corners of the matrix, rather than 2's in the original Rouse treatment.

Rouse's treatment for one molecule yields a discrete relaxation spectrum with $N$ lines of equal height $k T$, at relaxation times $\tau_{p}$, given by

$$
\tau_{p}=\left\langle r_{s}{ }^{2}\right\rangle\left(6 B k T \lambda_{p}\right)^{-1} \quad(p=1,2, \cdots, N),
$$

where $\lambda_{p}$ are the $N$ eigenvalues of $\mathbf{A}_{33}$, which are found to be

$$
\lambda_{p}=4 \sin ^{2}(p \pi / 2 N) \quad(p=1,2, \cdots, N) .
$$

The friction factor $1 / B$ is often written in a form which is $q$ times smaller, namely the monomeric friction co-

${ }_{13}^{13}$ J. A. Duiser and A. J. Staverman, in Physics of Non-Crystalline Solids, J. A. Prins, Ed. (North-Holland Publ. Co., Amsterdam, 1965), pp. 376-387. 
efficient $\zeta_{0}$ defined by

$$
Z \zeta_{0}=N / B
$$

where $Z$ is the degree of polymerization of the molecule.

The mobility coefficient matrix $\mathbf{A}_{33}$ thus controls the viscoelastic behavior of the molecule, since its eigenvalues determine the relative position of the relaxation times.

For a hypothetical molecule with fixed ends, ${ }^{13}$ the appropriate mobility coefficient matrix would be the square matrix

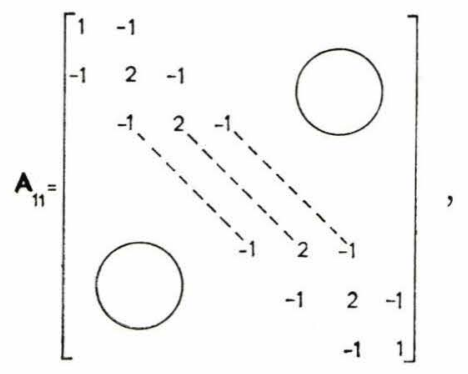

whose eigenvalues $\lambda_{p}$ are given by

$$
\lambda_{p}=4 \sin ^{2}(p \pi / 2 N) \quad(p=0,1,2, \cdots, N-1) .
$$

Equations (7) and (9) will be derived in a forthcoming article.

For $p<\frac{1}{5} N$ it is easily seen that $\lambda_{p} \approx p^{2} \pi^{2} / N^{2}$ or

$$
\tau_{p} \approx \tau_{1} / p^{2}
$$

where $\tau_{1}$ is the longest finite relaxation time of the molecule. By using Eq. (10) the discrete relaxation spectrum of a free molecule may be approximated by a continuous spectrum, which is found to be

$$
\begin{aligned}
H & =\frac{1}{2} k T\left(\tau_{1} / \tau\right)^{\frac{1}{2}} & & \left(\tau_{0,2 N}<\tau \leq \tau_{1} p_{0}^{-2}\right) \\
& =0 & & \left(\tau>\tau_{1} p_{0}^{-2}\right) .
\end{aligned}
$$

If the number $p_{0}$ is chosen to be about 0.64 a fair agreement is obtained between the dynamic moduli calculated from the continuous and the discrete spectra. Equation (9) differs from Eq. (7) only by the summation boundaries. Consequently, the relaxation spectrum of a molecule with fixed ends is equal to that of a free molecule except for one line at infinite relaxation time, corresponding to $p=0$.

\section{TWO-CHAIN PROBLEM}

In concentrated solutions, chain entanglement occurs provided the molecular weight is high enough. These entanglements are of a complicated geometrical nature. Molecules of polymers with very different degrees of chain stiffness caused by steric hindrance seem to become entangled to the same extent, ${ }^{3}$ indicating that an entanglement is not concentrated at one point on a chain. When devising a model, however, one has no choice but to concentrate the effect of entanglement coupling on infinitesimal points along the chain as is
Fig, 1. A two-chain network between four fixed points $\mathrm{A}, \mathrm{B}, \mathrm{C}$, and and $\mathrm{D}$, containing an entanglement at $\mathbf{P}$.

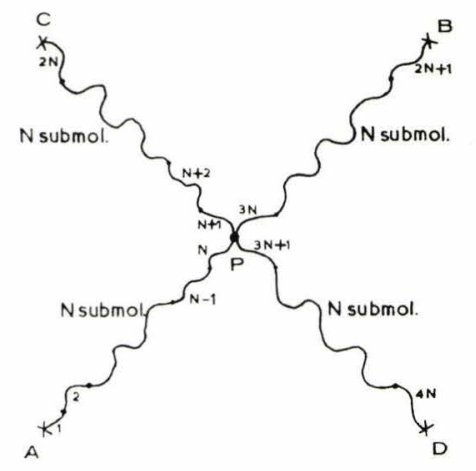

done in the model described below. The approximation does not seem serious, for theoretical and experimental results show a good fit. An essential requirement, however, is a large entanglement spacing along the molecule, i.e., the entanglement points should be separated from each other by several (at least two) submolecules. The model is therefore not applicable to every undiluted polymer system, but only to those polymer systems, preferably diluted, which yield a maximum rubbery shear modulus $G(t)$ or $G^{\prime}(\omega)$ of about $3 \times 10^{6}$ dyn $/ \mathrm{cm}^{2}$.

In the present treatment it is assumed that the effect of an entanglement is equivalent to a purely viscous coupling at the point of contact between two chains. The slipping of this coupling must be much slower than the movement of a free submolecule if it is to explain the presence of a group of long relaxation times beyond the glass transition region.

A two-chain network is considered, as shown in Fig. 1. $\mathrm{A}, \mathrm{B}, \mathrm{C}$, and $\mathrm{D}$ are fixed points and $\mathrm{P}$ is an entanglement point as described above, located halfway along $\mathrm{AC}$ and BD. Both chains contain $2 \mathrm{~N}$ submolecules, which are numbered as follows: from A to $\mathrm{P} ; 1$ to $N$, from $\mathrm{P}$ to $\mathrm{C}$; $N+1$ to $2 N$, from B to $\mathrm{P} ; 2 N+1$ to $3 N$ and from $\mathrm{P}$ to $\mathrm{D} ; 3 N+1$ to $4 N$. Following Rouse's method the average velocity of back diffusion along the $x_{N}$ and the $x_{3 N}$ coordinates is now calculated as follows. With all the junctions kept fixed except the $(N-1)$ th

$$
\left(\dot{x}_{N}\right)_{N-1}=-B\left[\left(\partial \mu / \partial x_{N}\right)-\left(\partial \mu / \partial x_{N-1}\right)\right] .
$$

With all the junctions kept fixed except the $N$ th

$$
\begin{aligned}
\left(\dot{x}_{N}\right)_{N}=-B\left\{\left(\partial \mu / \partial x_{N}\right)-\right. & \left(\partial \mu / \partial x_{N+1}\right) \\
& \left.+f\left[\left(\dot{x}_{N}\right)_{N}-\left(\dot{x}_{3 N}\right)_{3 N}\right]\right\},
\end{aligned}
$$

where $f\left[\left(\dot{x}_{N}\right)_{N}-\left(\dot{x}_{3 N}\right)_{3 N}\right]$ is the frictional force between the chains due to their velocity difference at $P$. The friction factor $f$ can have values from zero (for a free chain with fixed ends) to infinity (for a permanent crosslink).

Similarly, with all the junctions kept fixed except the $(3 N-1)$ th,

$$
\left(\dot{x}_{3 N}\right)_{3 N-1}=-B\left[\left(\partial \mu / \partial x_{3 N}\right)-\left(\partial \mu / x_{3 N-1}\right)\right]
$$


and with all the junctions kept fixed except the $3 N$ th,

$$
\begin{aligned}
\left(\dot{x}_{3 N}\right)_{3 N}=-B\left\{\left(\partial \mu / \partial x_{3 N}\right)\right. & -\left(\partial \mu / \partial x_{3 N+1}\right) \\
& \left.+f\left[\left(\dot{x}_{3 N}\right)_{3 N}-\left(\dot{x}_{N}\right)_{N}\right]\right\} .
\end{aligned}
$$

Combining Eqs. (13) and (15) gives

$$
\begin{array}{r}
\left(\dot{x}_{N}\right)_{N}=-B\left[\frac{1+B f}{1+2 B f}\left(\frac{\partial \mu}{\partial x_{N}}-\frac{\partial \mu}{\partial x_{N+1}}\right)\right. \\
\left.+\frac{B f}{1+2 B f}\left(\frac{\partial \mu}{\partial x_{3 N}}-\frac{\partial \mu}{\partial x_{3 N+1}}\right)\right], \\
\left(\dot{x}_{3 N}\right)_{3 N}=-B\left[\frac{1+B f}{1+2 B f}\left(\frac{\partial \mu}{\partial x_{3 N}}-\frac{\partial \mu}{\partial x_{3 N+1}}\right)\right. \\
\left.+\frac{B f}{1+2 B f}\left(\frac{\partial \mu}{\partial x_{N}}-\frac{\partial \mu}{\partial x_{N+1}}\right)\right] .
\end{array}
$$

Putting $[1 /(1+2 B f)]=\delta$, Eqs. (16) and (17) become

$$
\begin{aligned}
\left(\dot{x}_{N}\right)_{N}=-\frac{B}{2}[(1+\delta) & \left(\frac{\partial \mu}{\partial x_{N}}-\frac{\partial \mu}{\partial x_{N+1}}\right) \\
& \left.+(1-\delta)\left(\frac{\partial \mu}{\partial x_{3 N}}-\frac{\partial \mu}{\partial x_{3 N+1}}\right)\right],
\end{aligned}
$$

$$
\begin{aligned}
&\left(\dot{x}_{3 N}\right)_{3 N}=-\frac{B}{2}\left[(1+\delta)\left(\frac{\partial \mu}{\partial x_{3 N}}-\frac{\partial \mu}{\partial x_{3 N+1}}\right)\right. \\
&\left.+(1-\delta)\left(\frac{\partial \mu}{\partial x_{N}}-\frac{\partial \mu}{\partial x_{N+1}}\right)\right] .
\end{aligned}
$$

For crosslinked chains Duiser and Staverman ${ }^{13}$ have obtained equations which are equal to Eqs. (18) and (19) with $\delta=0$.

The factors $(1+\delta)$ and $(1-\delta)$ can be interpreted physically by noting that the mobility of each junction at $\mathrm{P}$ increases with a factor $(1+\delta)$ as the coupling becomes looser, while the force on it due to the junction belonging to the other chain decreases with a factor $(1-\delta) /(1+\delta) \approx(1-2 \delta)$ at the same time.

The quantity $\delta$ is thus a slip parameter which can have values from zero (permanent coupling) to unity (mutually free chains).

The equation for $\left(\dot{x}_{N}\right)_{D}$ can now be written by adding Eqs. (12) and (18). Similarly, the equation for $\left(\dot{x}_{3 N}\right)_{D}$ is obtained by adding Eqs. (14) and (19). The set of equations for all $\left(\dot{x}_{j}\right)_{D}$ can then be written

$$
\dot{\mathbf{x}}_{D}=-B \mathbf{A}_{e} \nabla_{x} \mu,
$$

where the new mobility coefficient matrix $\mathbf{A}_{e}$ is the square $4 N$ th-order matrix

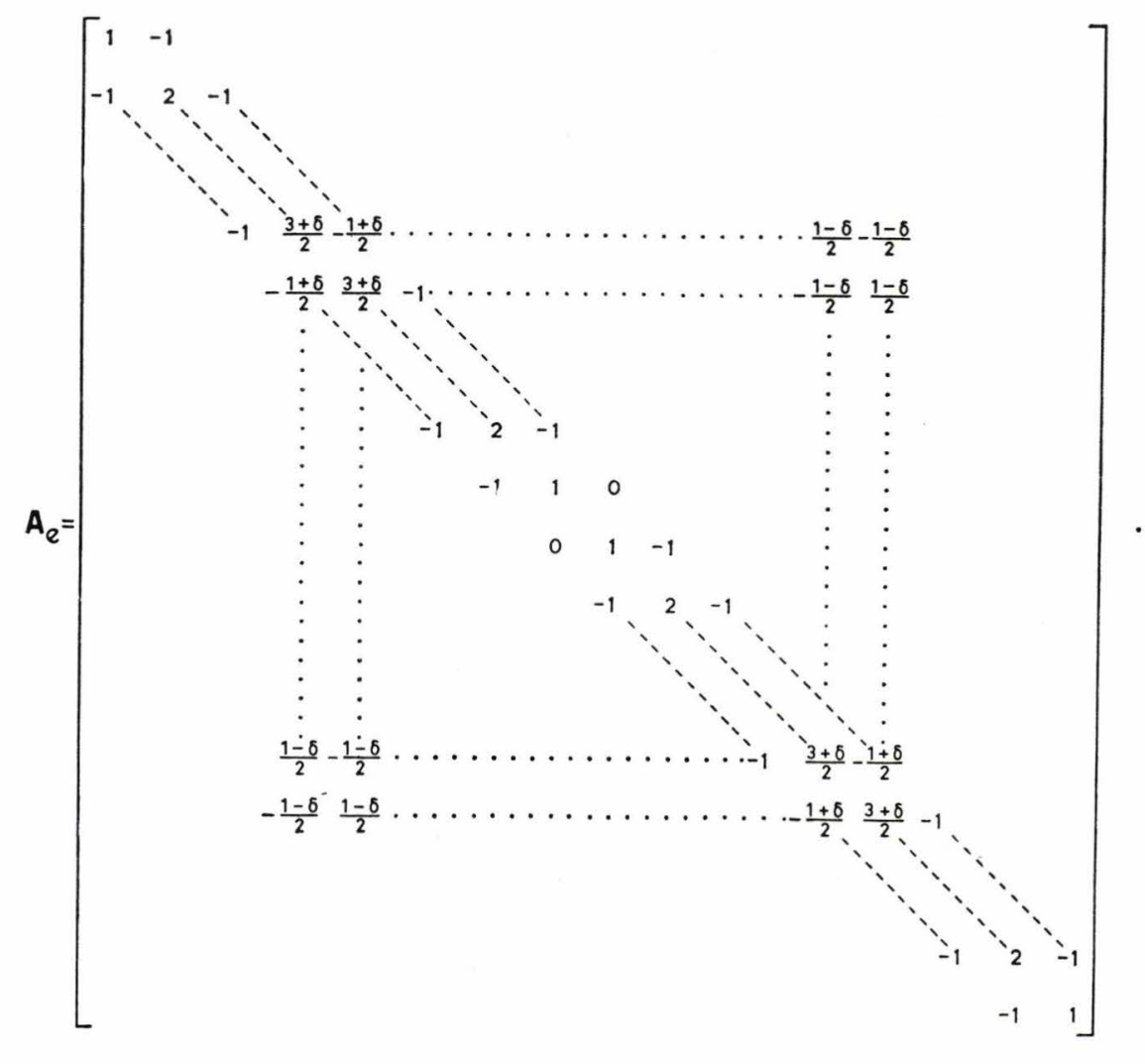


To find the eigenvalues of this matrix the characteristic equation $\left|\mathbf{A}_{e}-\lambda \mathbf{I}\right|=0$ can be simplified as follows: The $(2 N)$ th row is added to the $(2 N+1)$ th, the $(2 N-1)$ th row is added to the $(2 N+2)$ th, etc. Then the $(2 N+1)$ th column is subtracted from the $2 N$ th, the $(2 N+2)$ th column is subtracted from the $(2 N-1)$ th, etc. The resulting determinant can then be split into a product of two determinants, of which the first corresponds to a free chain of $2 \mathrm{~N}$ submolecules with fixed ends and the other corresponds to a chain with fixed ends and a point of increased friction (a "slow point") in the middle:

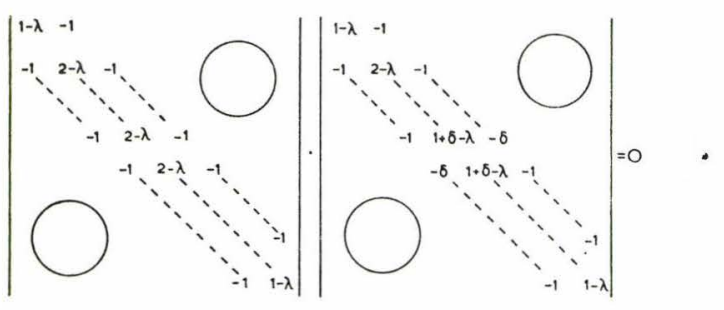

In accordance with the previous remarks, when $\delta$ goes to zero, the determinants of $\mathrm{Eq}$. (22) reduce to the result of Duiser and Staverman ${ }^{13}$ and become equivalent to one free chain with fixed ends, $2 \mathrm{~N}$ submolecules in length, and two free chains with fixed ends, each $N$ submolecules in length.

Thus the mathematical equivalence of the system of two chains between four fixed points, with one mobile entanglement, is as shown in Fig. 2. On the right-hand side one of the resulting chains (AC) is undisturbed, while the second (BD) of equal length possesses a lower mobility at the former point of contact $\mathrm{P}^{\prime}$. The mobility at such a "slow point" $\mathrm{P}^{\prime}$ is equal to $\delta B$. Physically $\delta B$ represents the ease of slipping of the entanglement point along one of the two chains.

\section{NETWORK PROBLEM}

The principle of the above mathematical equivalence can be applied to a whole network. To simplify the treatment it is assumed that the network may be represented as partly shown in Fig. 3(a). Initially, all the crosslinks are fixed points and the chains between them are all $N$ submolecules in length. For simplicity it is assumed that the mean-square end-to-end distance

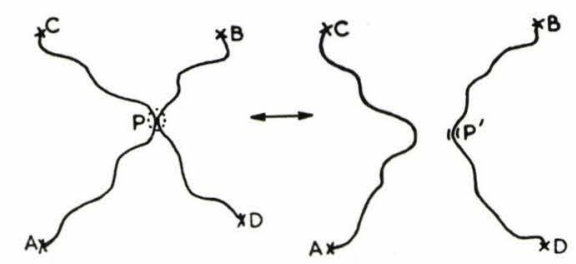

Fig. 2. The two-chain network and its mathematical equivalent consisting of two "decoupled" molecules, one of which carries a slow point $P^{\prime}$. between neighboring crosslinks is equal to that between two points on a free molecule separated by $N$ submolecules. In this case the front factor concerning the ratio of these distances may be omitted. The network is thought to be composed of $n$ primary molecules which are all $m N$ submolecules long; this indicates the presence of $(m-1)$ fixed crosslinks per primary molecule in this initial network. If half the crosslinks, indicated by a circle, are now transformed into entanglement points with a slip parameter $\delta$, then application of the above principle results in Stage $\boldsymbol{b}$. Again half of the remaining fixed crosslinks are successively transformed into entanglements giving Stage $c$, etc. Finally, only a few untransformed fixed points remain which lie on the outside boundaries of the network.

Thus an entanglement network consisting of $n$ molecules, each containing $(m-1)$ entanglements, may be transformed to its mathematical equivalent, which is a system of: $\frac{1}{4} n$ molecules with $(m-1)$ slow points, $+3 n / 8$ molecules with $\frac{1}{2}(m-1)$ slow points, $+3 n / 16$ molecules with $\frac{1}{4}(m-1)$ slow points, $+3 n / 32$ molecules with $\frac{1}{8}(m-1)$ slow points, etc. The relaxation spectrum per molecule of this entanglement network may now be written

$$
H_{\text {ent }}=\frac{1}{4} H[m-1]+\frac{3}{4} \sum_{k=1,2 \cdots}\left(1 / 2^{k}\right) H\left[(m-1) / 2^{k}\right],
$$

where $H[x]$ is the relaxation spectrum of a molecule ( $m N$ submolecules long) with $x$ slow points. The spectrum of each $H[x]$ can be obtained after solving the eigenvalues of the matrix $\mathbf{A}$, which contains $x$ slow points as shown in Eq. (24):

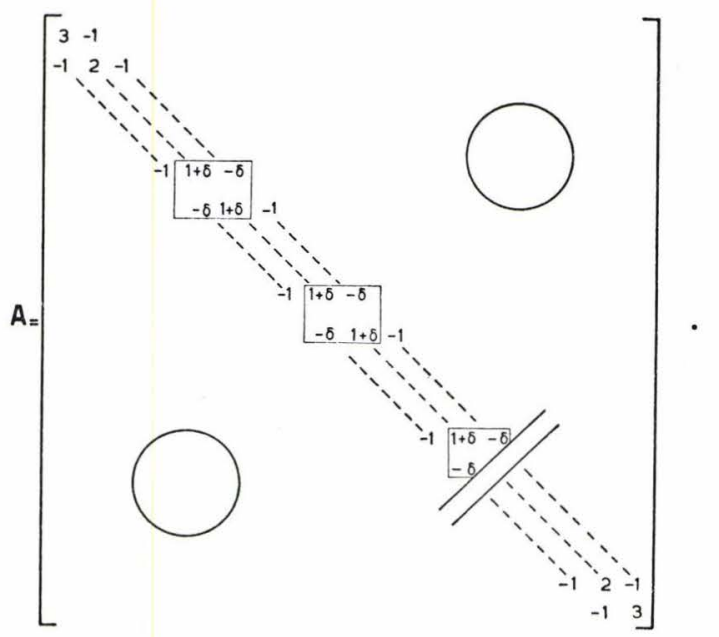

The square matrix $\mathbf{A}$ is always of the $m N$ th order.

The form of Eq. (21) indicates that the procedure for transformation of an entanglement network to the mathematically equivalent network, as in Fig. 2 or Eq. (22), is valid whenever the matrix (21) is symmetric about both its principal diagonal (i.e., each element $a_{i j}=a_{j i}$ ) and its other diagonal, i.e., each ele- 

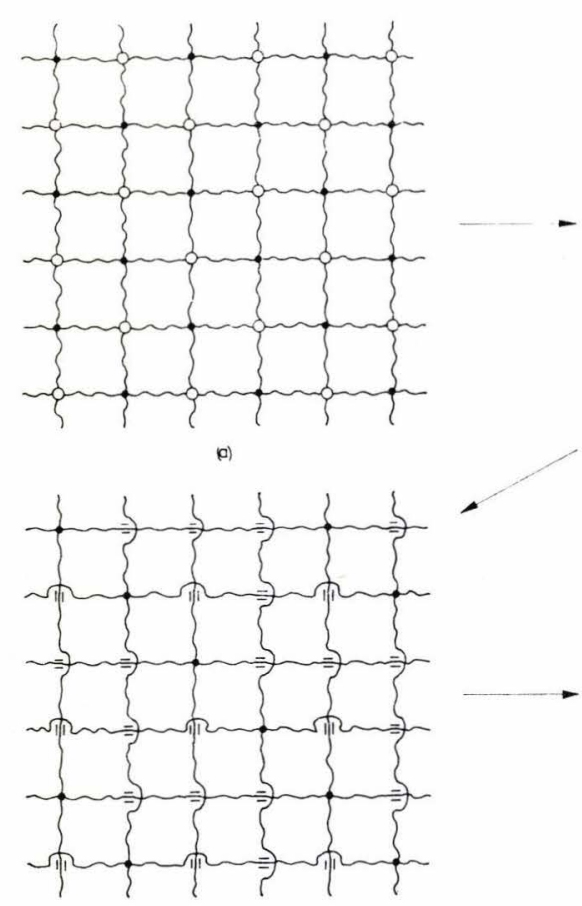

(c)

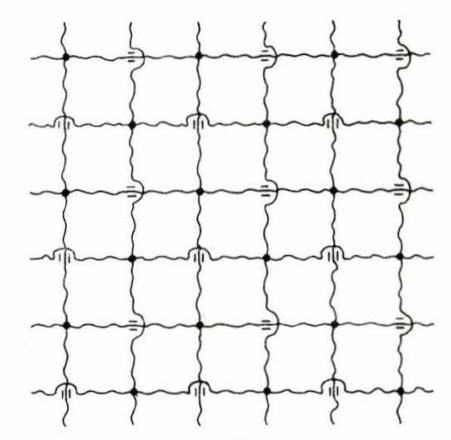

(b)

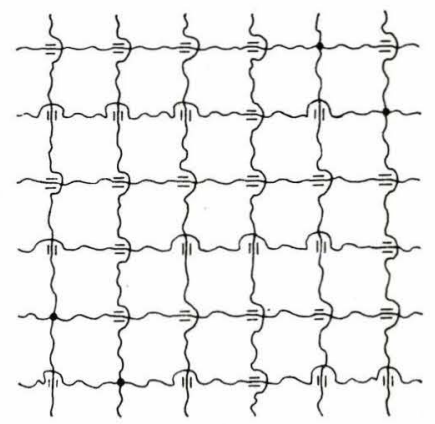

(d)
Fig. 3. The stepwise "decoupling" of chains in a regular network. ment $a_{i j}=a_{(N-j+1)(N-i+1)}$. This means that if two chains are entangled as shown in Fig. 4(a), where the chains are divided into two pairs of equal chain segments, they can be represented by their mathematical equivalence as in Fig. 4 (a).

This also means that a mathematical equivalence may be found for two entangling chains containing only two fixed points and two free ends as shown in Fig. 4(b), provided the entanglement divides the chains into two pairs of equal chain segments.

It has not yet been found possible to apply the above treatment to the general case where all four segments AP, BP, CP, and DP are different.

The physical meaning of these transformations can be illustrated as follows:

The chain AC can move with almost complete freedom. Once it has chosen a configuration, however, the point $\mathrm{P}$ appears to the chain $\mathrm{BD}$ as if it were fixed in

(a)
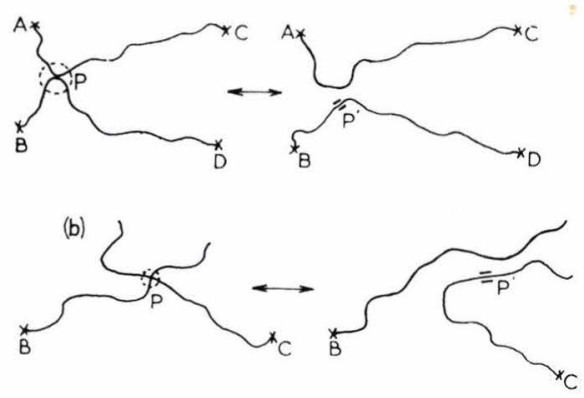

FIG. 4. The "decoupling" of somewhat irregularly entangled molecules. the case of a permanent crosslink, or as if it were a slow point in the case of an entanglement. The sections $\mathrm{BP}$ and $\mathrm{PD}$ then choose their own configuration independently. The system of four chain sections, therefore -instead of the commonly accepted four degrees of freedom in which elastic energy can be stored-has only three degrees of freedom in the case of a permanent crosslink and a variable number between 2 and 3 in the case of an entanglement depending on the time scale of the experiment.

There seems to be no obvious reason why this physical interpretation should not also apply to a case where $\mathrm{P}$ is not at the center of the chains $\mathrm{AC}$ and $\mathrm{BD}$, although it is not easy to prove this mathematically.

It is therefore assumed that the relaxation spectrum $H_{\text {ent }}$ of an entanglement network with random distribution of chain lengths between entanglements, is also represented by Eq. (23). Each term $H\left[(m-1) / 2^{k}\right]$ in this equation then represents an average of all possible entanglement distributions for a molecule with $(m-1) / 2^{k}$ slow points. In actual calculations, however, for each term $H\left[(m-1) / 2^{k}\right]$, one of the many possible distributions must be chosen and an average spectrum calculated from five to 10 such choices, depending on the accuracy which is desired.

\section{PERMANENTLY CROSSLINKED NETWORKS}

For the limiting case of a chemically crosslinked network where $\delta=0$, Eq. (23) may be simplified by writing the determinant of the characteristic equation of Eq. (24) as a product of smaller determinants. Then the relaxation spectrum $H_{\text {perm }}$ per elastically effective 
FIG. 5. The relaxation spectrum per network chain in a permanently crosslinked network.

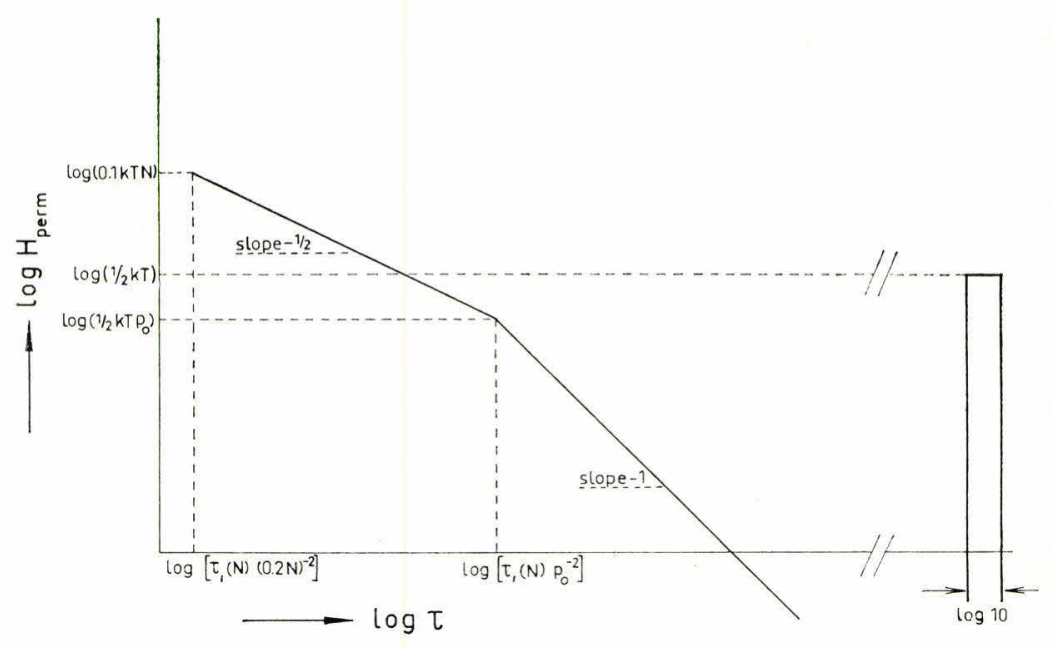

network chain is found to be

$$
H_{\text {perm }}=\frac{1}{4} H(N)+\frac{3}{4} \sum_{k=1,2 \cdots}^{\infty}\left(4^{k}\right)^{-1} H\left(2^{k} N\right),
$$

in which $H(y)$ is the relaxation spectrum of a chain with fixed ends and with a length of $y$ submolecules. It should be noted that the spectra represented by Eqs. (23) and (25) are no longer series of lines of equal height.

At infinite relaxation time, the summation of Eq. (25) can easily be carried out, because each $H(y)$ is associated with one mechanism with an infinite relaxation time. It is found that the total number of these mechanisms is half the number of elastically effective chains because

$$
\frac{1}{4}+\frac{3}{4} \sum_{k=1,2 \ldots}^{\infty}\left(4^{k}\right)^{-1}=\frac{1}{2} .
$$

As follows from Eqs. (6) and (7), the longest finite relaxation time of a chain is proportional to the square of the number of submolecules. So, if the longest finite relaxation time for a chain with $y$ submolecules is represented by $\tau_{1}(y)$, then

$$
\tau_{\mathbf{1}}\left(2^{k} N\right)=2^{2 k} \tau_{1}(N) .
$$

From Eq. (25) the following spectrum is obtained:

(a) At short times, where $\tau_{0,2 N}(N)<\tau \leq \tau_{1}(N) p_{0}{ }^{-2}$, substitution of Eqs. (11) and (26) in Eq. (25) and summation gives

$$
H_{\text {perm }}=\frac{1}{2} k T\left[\tau_{1}(N) / \tau\right]^{\frac{1}{2}},
$$

which is equal to $H$ of a free molecule, represented by Eq. (11).

(b) At longer times, where $\tau>\tau_{1}(N) p_{0}{ }^{-2}$, some terms of Eq. (25) become zero, as is shown by Eq. (11). A value $k_{0}$ may be defined in such a way, that only chains of $2^{k} N$ submolecules in length for which $k \geq k_{0}$ will contribute to the spectrum. Then in the region of the relaxation spectrum where

$$
\tau_{1}\left(2^{k_{0}-1} N\right) p_{0}{ }^{-2}<\tau \leq \tau_{1}\left(2^{k_{0}} N\right) p_{0}{ }^{-2}
$$

the summation of Eq. (25) must be carried out starting from $k_{0}$. With the use of Eq. (26), Eq. (28) can be written

$$
\frac{1}{4} 2^{2 k_{0}} \tau_{1}(N) p_{0}{ }^{-2}<\tau \leq 2^{2 k_{0}} \tau_{1}(N) p_{0}{ }^{-2} .
$$

So, the value of $k_{0}$ is related to $\tau$ in such a way that

$$
4 p_{0}{ }^{2} \tau / \tau_{1}(N)>2^{2 k_{0}} \geq p_{0}{ }^{2} \tau / \tau_{1}(N) .
$$

Defining a number $q_{0}^{2}$ lying between $\frac{1}{4}$ and 1 , one may write

$$
2^{2 k_{0}}=p_{0}^{2} \tau / q_{0}^{2} \tau_{1}(N) \text {. }
$$

Substitution of Eqs. (11) and (26) in Eq. (25) as before, but now carrying out the summation from a value $k_{0}$ determined by Eq. (31), yields

$$
\begin{aligned}
H_{\text {perm }} & =\frac{1}{2} k T\left[\frac{\tau_{1}(N)}{\tau}\right]^{\frac{1}{2}}{ }^{\frac{3}{4}} \sum_{k_{0}, k_{0}+1, \cdots}^{\infty} 2^{-k} \\
& =\frac{1}{2} k T\left[\frac{\tau_{1}(N)}{\tau}\right]^{\frac{1}{2}}\left(\frac{3}{4}\right) 2^{-k_{0}} \sum_{0,1,2 \ldots}^{\infty} 2^{-k} \\
& =\frac{1}{2} k T\left[\tau_{1}(N) / \tau\right]^{\frac{1}{2}}\left(\frac{3}{2}\right)\left[q_{0}{ }^{2} \tau_{1}(N) / p_{0}{ }^{2} \tau\right]^{\frac{1}{2}} \\
& =\frac{1}{2} k T\left[\tau_{1}(N) / \tau\right]\left(3 q_{0} / 2 p_{0}\right) .
\end{aligned}
$$

The value of $q_{0}$ is somewhat arbitrary; if it is taken to be $\frac{2}{3}$ the two parts of the spectrum derived in (a) and (b) will fit at $\tau=\tau_{1}(N) p_{0}^{-2}$.

The physical meaning of Eqs. (27) and (32) lies in the representation of two kinds of movements performed by the network. That part of the spectrum which is proportional to $\tau^{-\frac{1}{2}}$ [given by Eq. (27)] refers to movements of chains between neighboring crosslinks. At longer times it is followed by a part where the spectrum is proportional to $\tau^{-1}$ [given by Eq. (32)] corresponding to movements in which crosslinks participate. These movements may be extended over chain lengths larger than that of a primary molecule before crosslinking. Indeed extremely long relaxation times have been found experimentally. ${ }^{14}$ It should be empha-

\footnotetext{
${ }^{14}$ Reference 3, pp. 189 and 198.
} 
sized, however, that in a crosslinked polymer network additional entanglements also cause very long relaxation times. The theoretical relaxation spectrum per elastically effective network chain thus obtained, is illustrated in Fig. 5. The form of the peak at infinite time may be chosen arbitrarily provided the condition

$$
\int_{\text {peak }} H d \ln \tau=G_{e}=\frac{1}{2} k T
$$

is fulfilled. The theoretical relaxation spectrum per network chain thus obtained, is illustrated in Fig. 5.

\section{ENTANGLEMENT NETWORKS}

For the case of an entanglement network where $\delta$ is not equal to zero, Eq. (23) cannot be simplified further. To calculate the relaxation spectra of all the terms $H[x]$, a method has to be found to obtain the eigenvalues of a matrix like the one given in Eq. (24). Since the relaxation spectra obtainable from experiment are always continuous, the eigenvalues or consequently the relaxation times themselves do not have to be calculated, but only the density of relaxation times along the $\tau$ axis. This affords a short cut to the calculation of a continuous relaxation spectrum of each $H[x]$ by applying a property of the Sturm sequence. ${ }^{15}$

Moreover, random distribution of entanglement points over the entire molecule can now also be easily introduced.

The method is based on the fact that, given a tridiagonal determinant in the characteristic equation

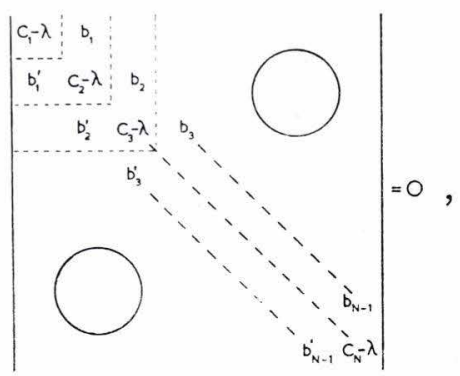

the subdeterminants $\left(H_{11}, H_{22} \cdots\right)$ indicated by dotted lines form the following sequence:

$$
\begin{aligned}
& H_{00}=+1, \\
& H_{11}=\left(c_{1}-\lambda\right) H_{00}, \\
& H_{22}=\left(c_{2}-\lambda\right) H_{11}-b_{1}{ }^{\prime} b_{1} H_{00}, \\
& H_{33}=\left(c_{3}-\lambda\right) H_{22}-b_{2}{ }^{\prime} b_{2} H_{11}, \\
& H_{i i}=\left(c_{i}-\lambda\right) H_{i-1, i-1}-b_{i-1}{ }^{\prime} b_{i-1} H_{i-2, i-2} .
\end{aligned}
$$

A theorem exists which proves that if a certain value of $\lambda$ is substituted into this "Sturm sequence," the number of agreements $\left(a_{l}\right)$ in sign between consecutive members of the sequence is equal to the number of

${ }^{15} \mathrm{~J}$. H. Wilkinson, Numerische Mathematik 4, 362 (1962). eigenvalues greater than that $\lambda$. If a $H_{i i}=0$ occurs, its sign is defined to be opposite to that of $H_{i-1, i-1}$.

For instance, for $\lambda=4$ in the matrix $\mathbf{A}$ of Eq. (24) all the $H_{i i}$ will alternate in sign, giving no eigenvalues larger than +4 . This maximum value for $\lambda$ of +4 follows obviously from Eq. (7) for $p=N$. For $\lambda=0$ all the $H_{i i}$ will be positive and thus all the eigenvalues will be larger than zero, as they should be.

At this point the lengthy calculations must be taken over by a computer, which is given the following instructions:

(1) Determine the number of agreements $a_{1}$ for a certain $\lambda_{1}$.

(2) Determine the number of agreements $a_{2}$ for $\lambda_{1}-\Delta \lambda_{1}=\lambda_{2}$ (where $\Delta$ is a multiplying factor, rather than an arithmetic increment, so as to give equal increments on a logarithmic scale).

(3) Find $a_{2}-a_{1}$ and repeat the process until $a_{l}=m N$, the order of the matrix.

(4) Give the results converted into $\log \tau_{R}$ values, where $\tau_{R}=1 / \lambda_{l}$.

As a result of such a calculation it was found that $\delta$ must be very small $\left(10^{-3}\right.$ to $\left.10^{-6}\right)$ in order to affect significantly the spectrum obtained with the unmodified Rouse matrix. For instance if $\delta=0.1$ the change is so slight as to be undetectable by experiment.

Clearly this result does not only apply to a chain with free ends, i.e., a matrix with 3 's in the corners, but also to a chain with fixed ends, i.e., a matrix with 1 's in the corners containing such perturbations as appear in Eq. (24). The results of these calculations will be given in a forthcoming article.

An approximate solution can be given for each term $H[x]$ of Eq. (23), provided $\delta$ is very small, by applying a method used by Kronig and Penney ${ }^{16}$ for the calculation of energy levels in a metal. A qualitative explanation for this approximation can be given as follows: At short times, where movements of chain segments occur which are much shorter than the average number of submolecules between entanglements, there is hardly any slip. The entanglements may then be considered as permanent crosslinks, or conversely the slow points may be considered as fixed points. This results in the type of relaxation spectrum discussed in the previous section. In the viscoelastic behavior at long times, however, the viscous properties of the molecule are mainly determined by the $x$ slow points, while the $(x+1)$ almost rubbery behaving chain segments between the slow points take care of the elastic properties of the molecule. In this long-time region the molecule may be considered as a series of slow points coupled by rubbery chain portions, which behave like giant "submolecules." The mobilities at the slow points are then taken as $\delta$ times the mobility of a normal submolecule.

${ }^{16}$ R. de L. Kronig and W. G. Penney, Proc. Roy. Soc. (London) A130, 499 (1931). 
FIG. 6. The approximate relaxation spectrum $H[m-1]$ for a "decoupled" molecule of $m N$ submolecules.

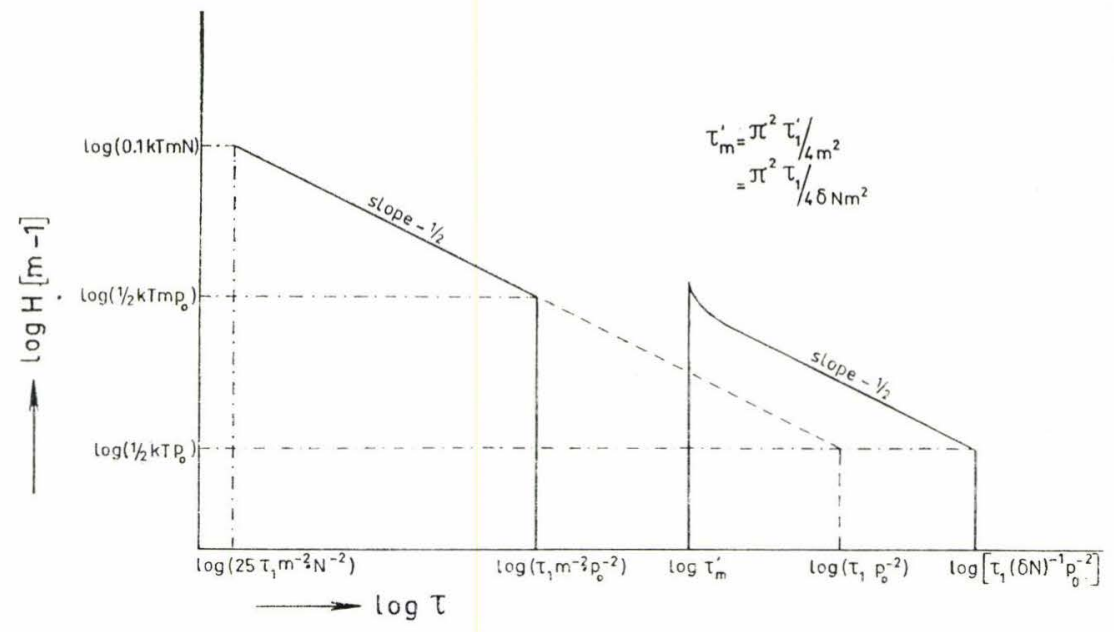

According to Eqs. (6) and (7) the relaxation times are inversily proportional to the mobilities at the junctions and are proportional to the square of the number of "submolecules" and to their average square end-toend distance. Thus, in the long-time region of $H[x]$ the approximation predicts a longest finite relaxation time $\tau_{1}^{\prime}$ of a molecule with $x$ slow points of

$$
\tau_{1}^{\prime}=\left\langle r_{e}^{2}\right\rangle(x+1)^{2} /\left(6 \pi^{2} k T \delta B\right),
$$

where $\left\langle r_{e}{ }^{2}\right\rangle$ is the average square end-to-end distance of the giant "submolecule" between slow points. The longest finite relaxation time of a molecule with the same length but without slow points is, according to Rouse,

Since

$$
\tau_{1}=\left\langle r_{s}{ }^{2}\right\rangle(m N)^{2} /\left(6 \pi^{2} k T B\right) .
$$

$$
\left\langle r_{e}^{2}\right\rangle=\left\langle r_{s}^{2}\right\rangle m N /(x+1),
$$

Eqs. (35) and (36) yield

$$
\left.\tau_{1}^{\prime} / \tau_{1}=(x+1) / m N \delta\right) .
$$

This result indicates that the very long-time region of the spectrum $H[x]$ should approximately correspond to a shift of part of the Rouse spectrum to higher values of $\tau$ by a factor of $(x+1) /(m N \delta)$. This is illustrated for the spectrum $H[m-1]$ in Fig. 6.

A similar type of relaxation spectrum has already been suggested intuitively by Ferry and co-workers ${ }^{17}$ for a real entangled molecule. In the present article, however, the spectrum of Fig. 6 is only valid for a molecule in the transformed equivalent system of molecules. The real spectrum $H_{\text {ent }}$ has to be assembled from a large number of such spectra according to Eq. (23).

Combining the results from the previous section for the short-time region with the above, yields a relaxation spectrum $H_{\text {ent }}$ of a shape as shown in Fig. 7.

The hump in the long-time region of the spectrum has a shape which is difficult to predict exactly, but the area under it is more important. Interpretation of the relaxation spectrum of Fig. 6 in terms of discrete line spectra, yields for $H[m-1]$ in the long-time region $(m-2)$ lines of equal height. Similarly, $H\left[\frac{1}{2}(m-1)\right]$ has in the long-time region $\frac{1}{2}(m-1)-1$ lines of equal height, and $H\left[\frac{1}{4}(m-1)\right]$ has in the same region

FIG. 7. Expected relaxation spectrum per molecule in an entanglement network.

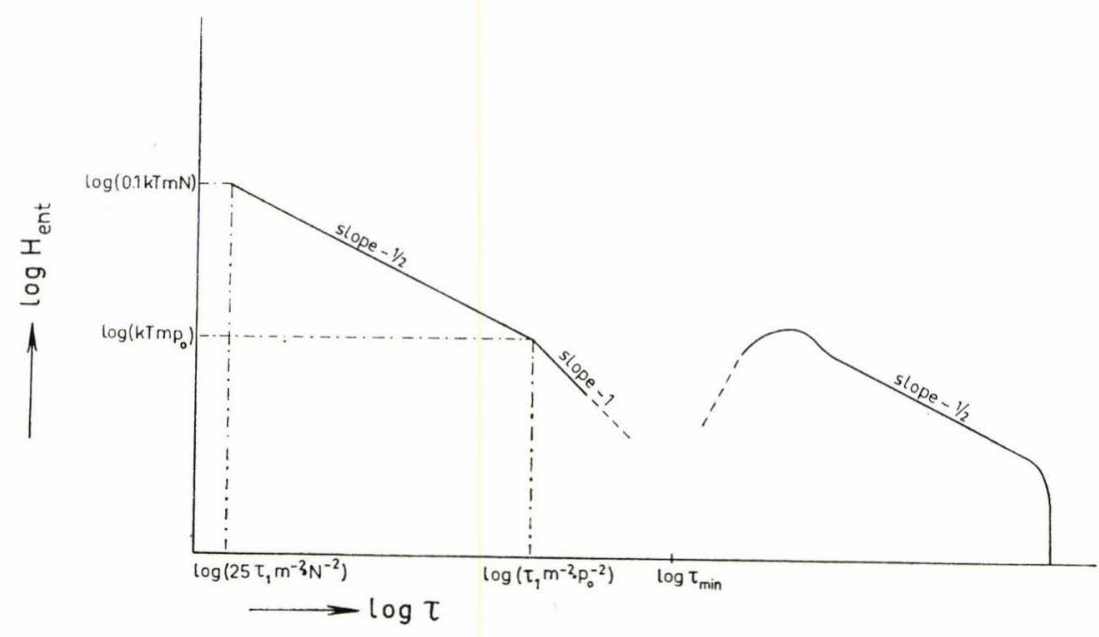

${ }^{17}$ J. D. Ferry, R. F. Landel, and M. L. Williams, J. Appl. Phys. 26, 359 (1955). 
$\frac{1}{4}(m-1)-1$ lines of equal height, etc. It is then obvious that the summation of all these lines in the long-time region, using Eq. (23), yields in Fig. 7 an area under the hump equal to half the number of elastically effective network chains $\left(\nu_{e}\right)$ multiplied by $k T$ because

$$
\begin{array}{r}
\frac{1}{4}(m-2)+\frac{3}{4} \sum_{k=1,2, \ldots}\left(1 / 2^{k}\right)\left\{\left[(m-1) / 2^{k}\right]-1\right\} \\
=\frac{1}{2}[(m-1)-2],
\end{array}
$$

which is exactly equal to half the number of elastically effective network chains per molecule.

Thus the long-time region of the relaxation spectrum of an entanglement network replaces the infinite-time region of a chemically crosslinked network (the "block" at infinite time in Fig. 5).

\section{CONCLUSIONS}

The theory of Rouse thus permits an extension which describes the viscoelastic relaxation spectrum of networks consisting of crosslinked or entangled macromolecules. The extension is performed by modification of the mobility-coefficient matrix in the configurationdiffusion equation, to give account of the frictional phenomena at an entanglement point between two entangled molecules.

It turns out that such a friction may be expressed mathematically by introducing a slip parameter $\delta$. Application of a decoupling process yields a system of two chains, where the mobility at the former point of contact on one of the two chains must be multiplied with this factor $\delta$, whereas the mobility at the same point on the other chain is kept unchanged.

Extension of this principle to a whole network yields also a mathematical equivalence, which is an assembly of decoupled molecules containing various amounts of "slow points." Since the relaxation spectra are assumed to be additive, the spectrum of the whole network can be obtained by appropriate summation of the different spectra of the decoupled molecules times their respective mole fractions.

A simplification can be achieved in the case where the molecules are permanently crosslinked $(\delta=0)$. Then the spectrum can be split into two regions: (1) At short times the slope of the logarithmic relaxation spectrum is $-\frac{1}{2}$, changing to -1 at a time corresponding to the longest relaxation time of a chain between two neighboring crosslinks; (2) at infinite time a peak in $H$ appears, corresponding to a number of mechanisms equal to half the number of elastically effective network chains. Both regions are illustrated in Fig. 5.

If $0<\delta<1$ the entanglements can be considered more or less similar to crosslinks. The long-time region of the relaxation spectrum no longer lies at infinite time. Instead, a hump in $H$ appears at fairly long relaxation times. The position of this hump depends on the value of $\delta$. Only for very low values of $\delta$ an approximate solution is applicable. Quite generally, however, the relaxation spectrum can be obtained by means of a computer using a property of the Sturm sequence. The relaxation spectrum thus obtained is schematically shown in Fig. 7. It has the following characteristic properties:

(a) In the short-time region the spectrum is identical with the spectrum for a crosslinked network, i.e., the slope of $-\frac{1}{2}$ is followed by a slope of -1 .

(b) A rather low minimum in the curve is followed by a hump, representing a group of long relaxation times. The integral of $H$ as a function of $\ln \tau$, corresponding to the hump, yields

$$
\int_{\min }^{\infty} H d \ln \tau=\frac{1}{2} \nu_{\epsilon} k T \text {. }
$$

Therefore, the number of elastically effective chains $\nu_{e}$, can be calculated directly from the spectrum derived from experiments. Examples of these calculations will be given in a forthcoming article.

(c) Beyond the maximum in the hump the slope is practically constant and equal to $-\frac{1}{2}$ up to the highest relaxation time. Relative to the short-time region where the slope is also equal to $-\frac{1}{2}$, this part of the spectrum may be considered as if the relaxation times of part of the Rouse spectrum have been shifted to higher values of $\tau$ over a distance proportional to $-\log (\delta N)$ along the time axis.

\section{ACKNOWLEDGMENTS}

The authors wish to thank Professor W. Prins and Professor A. J. Staverman for many helpful discussions throughout the course of this work and for the opportunity to conduct this work in their laboratories. The authors also wish to express their gratitude for the financial support from the Technische Hogeschool Delft, the University of Leiden, the Koninklijke/Shell Laboratorium Amsterdam, and the F.O.M. Scientific Research Council at Utrecht, all in The Netherlands. 